Mental Object Rotation and Motor Development in 8- and 10-Month-Old Infants

Andrea Frick and Wenke Möhring

University of Zurich

NOTICE: this is the author's version of a work that was accepted for publication in Journal of Experimental

Child Psychology. Changes resulting from the publishing process, such as peer review, editing, corrections, structural formatting, and other quality control mechanisms may not be reflected in this document.

Changes may have been made to this work since it was submitted for publication.

A definitive version is published as:

Frick, A., \& Möhring, W. (2013). Mental object rotation and motor development in 8- and 10-month-old infants. Journal of Experimental Child Psychology, 115, 708-720. DOI: 10.1016/j.jecp.2013.04.001

\title{
Acknowledgments
}

This research was supported by a research grant from the Swiss National Science Foundation \#PZ00P1_131866 to the first author. We thank Nora S. Newcombe for helpful comments on previous versions, Raquel Paz for her help with data collection and coding, Christian Paschke for his help with preparing the stimuli, and Henri Gossweiler for his help with producing the video sequences. Finally, we wish to thank the parents who kindly agreed to have their infants participate in this study. 


\begin{abstract}
Recent evidence indicates that 6-month-old infants' mental rotation of objects profits from prior manual experience, whereas observational experience does not have the same beneficial effect (Möhring, W. \& Frick, A., 2013, Child Development). The present study investigated whether older infants, at 8 and 10 months of age, succeed in this task after observational experience only, and whether performance is related to infants' motor development. Using the violation-of-expectation paradigm, infants $(N=40)$ were presented with an asymmetrical object that was moved straight down behind an occluder. After the occluder was lowered, infants saw either the original object (possible event) or a mirror image of the original object (impossible event) in one of five different orientations $\left(0^{\circ}\right.$ to $180^{\circ}$, in steps of $\left.45^{\circ}\right)$. Results indicated that it was not until 10 months of age that infants looked longer at the impossible outcome. Analyses including parent questionnaire data showed that mental rotation performance was related to infants' motor development emphasizing the importance of action experience for early cognitive development.
\end{abstract}

Keywords: mental rotation, cognitive development, spatial cognition, infants, motor development, embodied cognition 


\section{Mental Object Rotation and Motor Development in 8- and 10-Month-Old Infants}

Mental rotation is a well-examined spatial ability in human adults (e.g., Shepard \& Metzler, 1971). One reason for its importance in cognitive research is that evidence for mental rotation has been taken as a proof for the existence of depictive mental representations that are analogue to perception (Kosslyn, 1975). For example, findings in mental rotation research have shown that imagined movements reflect the same spatio-temporal characteristics as real movements, suggesting that they are subject to similar physical constraints as movements in the external world (Kosslyn, 1980). A second reason is that tests of mental rotation have often been used as markers for spatial abilities in adults' ability assessments and intelligence tests (for a review, see Hegarty \& Waller, 2005). However, despite the emphasis on mental rotation in adult research, the early origins of this particular ability and its individual differences are still unclear. The present study aimed to further explore the early development of mental rotation abilities in the first year of life, as well as the relation between motor development and infants' cognitive processing of rotational events.

The first studies to systematically investigate infants' understanding of rotational object movements presented 4- to 8-month-old infants with an object that rotated and moved on a curved trajectory and finally disappeared behind an occluder (Rochat \& Hespos, 1996; Hespos \& Rochat, 1997). When the occluder was lowered, the object was revealed in an orientation that was either consistent or inconsistent with the continued rotational movement. Results indicated that infants of all age groups looked longer at the inconsistent test event, suggesting that even the youngest infants were able to anticipate the outcome of the event. Recent studies on infants' mental object rotation presented infants from 3 to 5 months with asymmetrical objects in multiple views (Quinn \& Liben, 2008) or in motion, revolving through a $240^{\circ}$ angle (Moore \& Johnson, 2008, 2011). In subsequent test trials, infants' 
looking times towards the original object or its mirror image in previously unseen orientations were measured. Results showed that infants discriminated the original object from its mirror image in a novel orientation, suggesting that they mentally rotated the object.

The above studies left unanswered whether seeing the object in multiple views or in a rotational movement is crucial for infants' understanding of object rotation. This question was addressed by the following two studies: Möhring and Frick (2013) demonstrated that 6month-old infants were able to mentally rotate an object, even if they did not see a rotational trajectory before the object was occluded. Infants were presented with an asymmetrical object that was moved straight down behind an occluder. When the occluder was lowered, either the same object (possible event) or its mirror image (impossible event) was revealed in one of five different orientations (varying from $0^{\circ}$ to $180^{\circ}$, in steps of $45^{\circ}$ ). Results showed that infants looked longer at the impossible than at the possible outcomes. Importantly, 6-montholds succeeded in this task only if they had the opportunity to manually explore the test object prior to the experiment. In contrast, same-aged infants who were not allowed to touch the object did not differentiate between test events. In a similar vein but using a different approach, Frick and Wang (in press) demonstrated that 13- to 14-month-old infants were able to mentally track the orientation of an object on a turntable, even though the object was completely hidden during rotation. Again, 13- to 14-month-olds succeeded only after an exploration phase offering the opportunity to gather hands-on experience with the turntable carrying a different object. Taken together, these two studies showed that infants were able to mentally rotate objects even if they were not familiarized with a rotational movement of the test object. Moreover, these findings point to the importance of action experience in the form of direct manual exploration of the test object (Möhring \& Frick, 2013) or hands-on training with a turntable and a different object (Frick \& Wang, in press). Furthermore, there is recent correlational evidence that 9-month-old infants' crawling experience is associated with 
mental rotation performance (Schwarzer, Freitag, Buckel, \& Lofruthe, 2012) as measured by a task similar to the one of Moore and Johnson (2008).

These findings are in line with developmental theories positing that cognitive abilities are based on sensorimotor experiences (e.g., Piaget, 1936/1952; Piaget \& Inhelder, 1948/1956, 1966/1971). The idea of a close linkage between cognition and action or, in other words, between perceptual and motor systems of the brain, has recently gained new interest in the field of embodied cognition (Wilson, 2002; Zwaan, 1999). In support of this notion, recent evidence suggested that 4-year-olds' spatial transformation abilities were associated with how often they played with puzzles (Levine, Ratliff, Huttenlocher, \& Cannon, 2011). Further studies have shown that motor activities or motor constraints influenced children's and adults' mental object rotation (Frick, Daum, Walser, \& Mast, 2009; Funk, Brugger, \& Wilkening, 2005; Krüger \& Krist, 2009). Importantly, these studies point to a stronger influence of action experience in children than in adults, suggesting that the influence of action on cognition may change over the course of development. As they get older, children may become better at translating observed movements into covertly activated (but not executed) action plans (Wilson, 2002). Indeed, recent research showed that by 5 years of age, both manual and observational experience increased accuracy in a mental rotation task (Frick, Ferrara, \& Newcombe, 2013). In the present experiment, we investigated at which age infants would succeed at a mental rotation task after observational experience only. For this purpose, we used the same methodological approach as Möhring and Frick (2013), who showed that 6month-old infants did not succeed after observational experience only, and tested older infants at the age of 8 and 10 months.

Assuming that the ability to learn from observational experience increases with age, this raises the question of what kinds of mechanisms promote this development. A possible scenario is that maturation of infants' own motor abilities has a positive effect on how much 
information they can gain through observation. Manipulating objects or moving among them may provide infants with a range of opportunities to view objects in different orientations and from different perspectives. Indeed, previous research provided evidence for a relation between locomotor development and infants' spatial cognition in general (see Campos et al., 2000 for a review), and mental object rotation in particular (Schwarzer et al., 2012).

Therefore, infants' motor development was assessed by means of a parent questionnaire in the current study.

\section{Method}

\section{Participants}

Twenty healthy and full-term infants at the mean age of 8 months and 2 days $(S D=8$ days; range: 7 months, 17 days to 8 months, 15 days; 10 girls) and twenty infants at the mean age of 10 months and 21 days $(S D=20$ days; range: 9 months, 18 days to 11 months, 14 days; 10 girls) participated. For the sake of simplicity, these age groups will subsequently be referred to as 8 - and 10-month-olds.

Four additional infants were tested but excluded from the sample due to fussiness (2) and failure to pass the familiarization criterion (2). According to this criterion, infants were excluded if they had looked less than the duration of one event presentation (less than $5.8 \mathrm{~s}$ ) on two of three familiarization trials, to ensure they had a chance to become familiar with the general pattern of the events. Infants were predominantly Caucasian, from middle-class backgrounds, and lived in urban and suburban areas of a large Swiss city. Parents filled out a consent form and a questionnaire on their infants' motor development prior to the study. Infants received a small toy and a certificate for their participation after the study.

\section{Stimuli}

In the familiarization trials, infants were presented with a symmetrical object in the shape of the letter "T". The T-object was made of plywood (10 cm high x $7.5 \mathrm{~cm}$ wide) and 
painted blue. In the test trials, infants were presented with two asymmetrical objects that had the shapes of a "p" and a "q" (see Figure 1). Both were made of plywood, and painted blue in front (10 cm high x $5 \mathrm{~cm}$ wide). The backs of the objects were constructed to look and feel very different compared to the front sides: five concentric plywood circles of decreasing diameter, painted alternatingly in red and yellow, were glued onto the yellow backs of the objects. Thus, the two objects were mirror objects that could not be superimposed onto each other by rotation about any axis.

In order to create video sequences for the familiarization and test trials, the T-, $\mathrm{p}$ - and q-objects were filmed against a wooden backboard $(66 \mathrm{~cm}$ high $\mathrm{x} 100 \mathrm{~cm}$ wide) that was painted white; however, some of its wooden structure was still visible. An opening $(20 \mathrm{~cm}$ high $\times 20 \mathrm{~cm}$ wide) at the center of the bottom edge of the backboard allowed an experimenter to reach through and move the objects with her right arm. An invisible glass pane was mounted parallel in front of the backboard and aided the experimenter in holding the object steadily. At the beginning of each trial (except for the second and third familiarization trial) a gray occluder $(21 \mathrm{~cm}$ wide $\times 21 \mathrm{~cm}$ high $)$, placed parallel in front of the glass pane, completely covered the view of the smaller cut-out in the backboard.

\section{Procedure}

In an initial encoding phase, infants had the opportunity to gather visual information about the test object that they would later see disappear behind the occluder (i.e., p or q, depending on condition). In a live presentation, an experimenter moved the object in front of the infants, rotating it about its vertical axis ( $180^{\circ}$ back and forth, for 2 minutes), in order to show that the object's back and front looked very different. However, infants were not allowed to touch the object during this kind of presentation. A Plexiglas window $(75 \mathrm{~cm}$ high $\mathrm{x} 50 \mathrm{~cm}$ wide), attached to the table prevented them from grasping the object. 
Immediately after the encoding phase, infants' mental rotation abilities were tested using the violation-of-expectation paradigm. Infants sat on the parent's lap approximately 60 $\mathrm{cm}$ in front of a 30-inch TFT computer screen. Dark brown curtains, extending from the ceiling to the floor, fully enclosed the viewing area. A small hole $3.5 \mathrm{~cm}$ above the computer screen allowed for a video camera to capture infants' looking behavior. Each trial began with an attention getter (rapidly alternating geometric shapes) that was presented on the upper part of the computer screen, at the position where the familiarization and test objects would later appear. Once the infant looked at the attention getter, the experimenter started the trial and looking time was recorded. Familiarization and test trials ended when the infant looked away for 2 consecutive seconds or when 60 seconds had elapsed.

Infants' looking times were measured online by the experimenter. In order to calculate inter-rater reliability, videos of ten randomly chosen infants of each age group were coded off-line by a second naïve experimenter. The average Pearson correlation of looking times during test trials was $r=.94$ for the 8 -month-olds and $r=.96$ for the 10 -month-olds.

\section{Events}

Events were short video sequences that were edited by means of the program Adobe Premiere Pro CS3. First, infants were presented with three familiarization events and then saw ten test events. Each familiarization event lasted a total of $8.8 \mathrm{~s}$ and was shown repeatedly, with a black screen of $1.2 \mathrm{~s}$ between each repetition. In the first familiarization event, the experimenter presented the upright T-object at the top of the screen $(1 \mathrm{~s})$. Next, she moved the T-object down vertically by $30 \mathrm{~cm}(3 \mathrm{~s})$, where it disappeared behind the occluder, and continued until it reached the middle of the occluder $(0.5 \mathrm{~s})$. After a short time interval (1 s), the occluder was lowered $(0.3 \mathrm{~s})$, and the object was presented ( $3 \mathrm{~s}$, freeze frame). In the first familiarization trial, the object was not rotated. In the second familiarization event, the occluder was absent. Thus, infants were able to see the entire trajectory of the object's 
movement. Analogous to the first familiarization event, the T-object was held steadily ( $1 \mathrm{~s})$ and moved straight down (3.5 s). At the point where the object would have reached the middle of the occluder, the hand turned the object $30^{\circ}$ clockwise $(1 \mathrm{~s})$ and then held it in this final orientation $(3.3 \mathrm{~s})$. The third familiarization event was identical to the second, except that the object was rotated $150^{\circ}$ clockwise $(1 \mathrm{~s})$. The purpose of the familiarization was to accustom infants to the occlusion event, the straight-down movement, as well as the rotational movement. Crucially, rotational movements were only shown using a different object and different rotation angles than those presented in subsequent test events.

The test events followed the same event structure as the first familiarization event, but differed in the objects used and the outcomes infants saw after the occluder was lowered (see Figure 2). In the test events, the p-object (or the q-object) was presented at the top of the screen showing its blue side only ( $1 \mathrm{~s})$. The object was moved straight down (3 s), and disappeared behind the occluder. After enough time for the experimenter to move the object to the midpoint of the occluder $(0.5 \mathrm{~s})$ and to rotate it $(1 \mathrm{~s})$, the occluder was lowered $(0.3 \mathrm{~s})$. This revealed either the original object (possible event) or its mirror version (impossible event) in one of five different orientations (in the picture plane, thus still showing their blue sides). Infants watched the final paused scene with the object remaining in its outcome orientation. The beginning of the event as well as the lowering of the occluder was marked by a ding sound (Windows Media ding.wav) in order to attract infants' attention. Because the backs of the objects were different and the objects could not be brought into congruence by rotation about any axis, infants' expectation of object consistency should be violated by the impossible outcome, resulting in their longer looking times.

\section{Design}

Each participant saw ten test trials that varied in type of test event (possible, impossible) and outcome orientation $\left(0^{\circ}, 45^{\circ}, 90^{\circ}, 135^{\circ}, 180^{\circ}\right.$ in picture plane). Possible and 
impossible events of each orientation were paired and presented one after the other. However, it was counterbalanced between participants in which order they saw the possible (p) and impossible (i) events: pi ip ip pi ip - or - ip pi pi ip pi. In addition, the order in which the five outcome orientations were presented was counterbalanced between participants according to a Latin-square design. It was held constant within participants, and was counterbalanced between participants, whether they always saw the q- or p-object disappearing behind the occluder. This resulted in 20 different combinations, each of which was randomly assigned to one participant of each age group.

\section{Parent questionnaire}

Parents were asked to fill in a questionnaire about their infants' motor development. The questionnaire was developed for the present study and consisted of questions about infants' object manipulation and locomotion. For example, parents were asked whether their child was able to crawl with its belly touching the floor, crawl on hands and knees, walk with assistance, or walk freely, and since when their child showed this behavior (see Appendix A). Items were scored in number of months (including half months) infants had experience with the particular motor ability.

\section{Results}

\section{Familiarization}

Looking times during the familiarization events were compared using an ANOVA with age group as between-subjects variable. No main effect of age group, $F(1,38)=0.21, p$ $=.65, \eta^{2}=.01$, and no interaction of age group and familiarization event were observed, $F<$ 1, suggesting that 8 - and 10-month-olds did not differ in their looking behavior during familiarization.

\section{Test events}


The following results are based on infants' looking times after the occluder was lowered and the final outcome of the test event was revealed. A preliminary overall analysis of variance (ANOVA) revealed no main effects of order of test events (possible vs. impossible first) and type of disappearing object ( $p$ or q), or interactions with the withinsubject variables of interest, all $F \mathrm{~s}<2.33$, all $p \mathrm{~s}>.14$ (except for two four-way-interactions that were not interpretable). Thus, these variables were not considered in the following analyses.

An ANOVA with the between-subjects variables of age and sex and the withinsubject variables of test event (possible vs. impossible) and orientation $\left(0^{\circ}, 45^{\circ}, 90^{\circ}, 135^{\circ}\right.$, $180^{\circ}$ ) yielded significant main effects of age group, $F(1,36)=10.24, p<.01, \eta^{2}=.22$, and of test event, $F(1,36)=9.90, p<.01, \eta^{2}=.22$. Overall, infants looked longer at the impossible than at the possible test events, and 10-month-olds looked longer at the test events than 8month-olds. However, these main effects were qualified by a significant interaction between test event and age group, $F(1,36)=6.94, p<.05, \eta^{2}=.16$ (see Figure 3). Pairwise comparisons (Bonferroni corrected) showed that 10-month-olds looked significantly longer at the impossible event $(M=8.11, S E=0.75)$ than at the possible event $(M=5.36, S E=0.44), p$ $<.001$, whereas the 8-month-olds looked equally at the impossible $(M=4.54, S E=0.75)$ and possible $(M=4.30, S E=0.44)$ test events, $p=.72$.

Furthermore, the analysis showed a significant main effect of sex, $F(1,36)=6.41, p<$ $.05, \eta^{2}=.15$, due to girls looking longer at the test events $(M=6.49, S E=0.51)$ than boys $(M$ $=4.66, S E=0.51)$. However, girls and boys did not differ regarding their looking times at the possible and impossible events, $F(1,36)=2.32, p=.14, \eta^{2}=.06$. There were no other significant effects and, in particular, no effects or interactions of orientation, all $F_{\mathrm{S}}<1.86$, all $p \mathrm{~s}>.12$, showing that the discrimination of the possible and impossible event outcomes was not affected by the angle of rotation (see Figure 4). 
The difference in the age groups' looking behaviors toward the test events was confirmed by a non-parametric test (Fisher's exact test, two-sided), comparing the number of infants in each age group who looked longer at the impossible than at the possible test events (averaged across all impossible and possible events). Results showed that significantly more 10-month-olds (18 out of 20 infants) looked longer at the impossible events compared to 8month-olds (9 out of 20 infants), $p<.01$.

\section{Encoding phase}

To find out whether 10-month-old infants' preference for the impossible test event was due to a quantitatively longer encoding of the asymmetrical object during the encoding phase, an independent samples $t$-test was calculated. However, eight-month-old infants looked significantly longer at the presented object $(M=82.04, S D=18.97$, ranging from 36.02 to $109.18 \mathrm{~s})$ than 10 -month-olds $(M=65.98, S D=12.65$, ranging from 43.21 to 89.04 s), $t(38)=3.15, p<.01, d=.996$.

\section{Motor development}

In a first step, it was investigated which of the variables assessed by the parent questionnaire was associated with performance in the mental rotation task. For that end, a difference score was calculated by subtracting the mean looking times during possible trials from the mean looking times during impossible trials. Thus, large difference scores indicate that infants differentiated between the possible and impossible outcomes, and positive scores indicate longer looking at the impossible test events. Spearman correlations revealed that this difference score was positively related to the time (in months and half months) infants had experience with a number of manual abilities, such as grasping, turning, or tilting objects, as well as some gross-motor abilities, such as crawling, standing, or walking with assistance (at $p<.05$, Spearman's Rho ranging from .33 to .49 , see Table 1). A second analysis showed that, after controlling for age, mental rotation performance remained significantly correlated 
to tilting a glass, and to standing up, standing, and walking with assistance (at $p<.05$; partial Spearman's Rho ranging from .36 to .40$)$. Overall, the strongest correlations were found for gross-motor abilities that are relevant for self-locomotion.

To further explore effects of infants' self-locomotion experience on their mental rotation performance, infants of both age groups were assigned to four categories reflecting their locomotion abilities: pre-locomotor $(n=8)$, belly crawling $(n=8)$, crawling $(n=12)$, and walking with assistance $(n=12)$. An ANOVA was calculated with test event (possible vs. impossible) as within-subject variable, locomotor experience (pre-locomotor, bellycrawling, crawling, walking with assistance) as between-subjects variable, and looking times as dependent variable. The analysis yielded no main effect of locomotor experience, $F(3,36)$ $=1.20, p=.32, \eta^{2}=.09$, but a significant main effect of event $F(1,36)=7.00, p<.05, \eta^{2}=$ .16. More importantly, the analysis revealed a significant interaction between test event and locomotor experience, $F(3,36)=3.19, p<.05, \eta^{2}=.21$. Pairwise comparisons (Bonferroni corrected) revealed that infants who were walking with assistance looked significantly longer at the impossible $(M=8.30, S E=1.09)$ than at the possible events $(M=4.75, S E=0.61), p<$ .001 , whereas infants with other or no locomotion experience did not differ in their looking times toward the test events, all $p \mathrm{~s}>.23$ (see Figure 5). In an analogous analysis with age as covariate, the interaction between test event and locomotor experience did not reach statistical significance, $F(3,35)=2.11, p=.12, \eta^{2}=.15$. This may have been due to a strong correlation between age and locomotor experience (Spearmans' $\mathrm{Rho}=.54, p<.001$ ).

\section{Discussion}

The present study was based on previous work (Möhring \& Frick, 2013) that provided evidence for 6-month-old infants' mental object rotation after hands-on experience with the test object. Conversely, same-aged infants who received observational information only did not discriminate between possible and impossible test events, suggesting that at this early age 
action experience is crucial for infants' mental object rotation. The present findings extend these results in various ways. They showed that it was not until the age of 10 months that infants became able to succeed in the same mental rotation task after gathering observational experience only. Ten-month-old infants looked significantly longer at the impossible than at the possible test events, whereas 8-month-olds did not distinguish between the events. Thus, it appears that only infants of the older age group were able to mentally rotate the object from its vertical starting position and recognized that a new object was presented after the hidden rotation in the impossible test events.

One possible explanation for the observed difference between the age groups could be that infants at the age of 8 months were not able to gather sufficient visual information about the test object during encoding or about the general event structure during familiarization. However, results showed that 8-month-old infants looked significantly longer toward the object during the encoding phase compared to 10-month-olds. Nevertheless, 10-month-olds were more apt to make use of the provided visual information, indicating that their encoding was more efficient. Moreover, the two age groups did not differ in their looking times during familiarization trials, ruling out the possibility that differences in familiarization could have accounted for the observed age difference in test trials.

Another explanation for the observed age differences between 8 and 10 months could be gained from analyzing information on infants' motor development. Analyses of parent questionnaire responses revealed positive correlations between infants' motor experience (especially tilting a glass or standing up, standing, and walking with assistance) and their individual inclinations to look longer at the impossible than at the possible events, even after controlling for age. This result suggests that infants' motor development seemed to play an important role for their ability to realize that a new object was presented in the impossible test events, likely mediating the age effect between 8- and 10-month-olds. 
Although correlations were found for a number of motor abilities, the strongest correlation was found between infants' mental rotation performance and their walking with assistance. This result extends previous findings (Schwarzer et al., 2012) and raises the question of why locomotor experience seems to be so closely linked to mental object transformations. On the one hand, it is conceivable that increasing experience with selfinitiated changes in perspectives enables infants to think about space in more allocentric terms (Needham \& Libertus, 2011). That is, their reasoning about spatial relations between objects (or objects and agents) may become increasingly independent from their own location and perspective. Indeed, there is evidence that the onset of independent locomotion has a strong influence on a variety of cognitive (spatial) as well as social and emotional abilities (for a review see Campos et al., 2000). On the other hand, walking skills may be an indicator of motor development in general. That is, infants who are early walkers may be generally more physically advanced as compared to non-walkers, which opens up more opportunities to explore objects and their spatial environment.

In this respect, it has to be noted that parents filled out the motor development questionnaire retrospectively. Thus, they might have been especially accurate in providing information for their infants' walking behavior as it was (a) one of the more recently achieved abilities and (b) a very salient ability that naturally many parents take note of. Therefore, it could have been that this variable was least affected by memory distortions, and thus proved to be the strongest correlation in our analyses due to its low error variance. Conversely, the non-significant correlations in our results could be due to the fact that some less salient motor development (e.g., precision grip) might have gone unnoticed by the parents, and therefore should not be over-interpreted. Future studies that assess motor development more directly using longitudinal approaches may clarify the role of other less salient motor abilities. 
An alternative analysis (ANOVA) confirmed that infants with more developed locomotor abilities were better able to differentiate between test events. However, a followup analysis including age as a covariate did not reveal significant results. This suggests that locomotor ability cannot be totally separated from age. However, these non-significant results may also be due to collinearity as the two variables were significantly correlated.

Nevertheless, the fact that the correlations reported above remained significant even when controlling for age suggests an association of locomotor abilities with mental rotation performance above and beyond age.

The result that in the present task 8-month-olds did not discriminate between test events seems to contradict previous findings of 3- to 5-month-old boys discriminating between original objects and their mirror versions (Moore \& Johnson, 2008, 2011; Quinn \& Liben, 2008). However, it is possible that methodological differences accounted for this divergence. In the present task, infants never saw the test object in any other orientation but upright. In contrast, previous studies presented infants with a large amount of the object's rotational movement (Hespos \& Rochat, 1997; Moore \& Johnson, 2008, 2011; Rochat \& Hespos, 1996), or with the object in various orientations (Quinn \& Liben, 2008) prior to the test. It is possible that recognizing an object in a novel orientation is easier if multiple familiar views of an object can be interpolated or a presented movement can be extrapolated, as opposed to recognizing an object rotated in a completely novel plane (cf., Bülthoff \& Edelman, 1992). Therefore, the present task was probably more difficult for young infants, but can be viewed as a stronger and more conclusive test of infants' mental rotation ability.

In conclusion, the results of the present study have several major implications. First of all, they indicate that in a mental rotation task that requires a rotation in an novel plane, and thus cannot be solved by inter- or extrapolating familiar views, it was not until 10 months of age that infants succeeded without manually encoding the test object. Second, these results 
suggest that unlike 8-month-olds, 10-month-olds are able to learn from observation in order to inform their cognitive processing of objects and events. Third, the present findings suggest that motor development is associated with infants' mental rotation performance. Hence, the present results support theories of embodied cognition proposing a close linkage between action and cognition (e.g., Wilson, 2002, Zwaan, 1999), as well as theories highlighting the pivotal role of sensorimotor experience in cognitive development (e.g., Piaget, 1936/1952; Piaget \& Inhelder, 1948/1956, 1966/1971). This indicates that motor development deserves more focused attention in future studies. Investigating factors that affect the early development of spatial abilities may help to reduce individual differences that may impede full participation in a technological society later in life (Newcombe \& Frick, 2010). 


\section{References}

Bülthoff, H., \& Edelman, S. (1992). Psychophysical support for a two-dimensional view interpolation theory of object recognition. Proceedings of the National Academy of Sciences of the United States of America, 89, 60-64.

Campos, J. J., Anderson, D. I., Barbu-Roth, M. A., Hubbard, E. M., Hertenstein, M. J., \& Witherington, D. (2000). Travel broadens the mind. Infancy, 1, 149-219.

Frick, A., Daum, M. M., Walser, S., \& Mast, F. W. (2009). Motor processes in children's mental rotation. Journal of Cognition and Development, 10, 18-40.

Frick, A., Ferrara, K., \& Newcombe, N. S. (2013). Using a touch screen paradigm to assess the development of mental rotation between $3 \frac{1}{2}$ and $5 \frac{1}{2}$ years of age. Cognitive Processing. DOI: 10.1007/s10339-012-0534-0.

Frick, A., \& Wang, S. (in press). Mental spatial transformations in 14- and 16-month-old infants: Effects of action and observational experience. Child Development.

Funk, M., Brugger, P., \& Wilkening, F. (2005). Motor processes in children's imagery: The case of mental rotation of hands. Developmental Science, 8, 402-408.

Hegarty, M., \& Waller, D. (2005). Individual differences in spatial abilities. In P. Shah, \& A. Miyake (Eds.), The Cambridge handbook of visuospatial thinking (pp. 121-169). Cambridge University Press.

Hespos, S. J., \& Rochat, P. (1997). Dynamic mental representation in infancy. Cognition, 64, 153-188.

Kosslyn, S. M. (1975). Information representation in visual images. Cognitive Psychology, 7, 341-370.

Kosslyn, S. M. (1980). Image and mind. Cambridge, MA: Harvard University Press. 
Krüger, M., \& Krist, H. (2009). Imagery and motor processes - when are they connected? The mental transformation of body parts in development. Journal of Cognition and Development, 10, 239-261.

Levine, S. C., Ratliff, K. R., Huttenlocher, J., \& Cannon, J. (2011). Early puzzle play: A predictor of preschoolers' spatial transformation skill. Developmental Psychology, 48, $530-542$.

Möhring, W., \& Frick, A. (2013). Touching up mental rotation: Effects of manual experience on 6-month-old infants' mental object rotation. Child Development. DOI: 10.1111/cdev.12065.

Moore, D. S., \& Johnson, S. P. (2008). Mental rotation in human infants: A sex difference. Psychological Science, 19, 1063-1066.

Moore, D. S., \& Johnson, S. P. (2011). Mental rotation of dynamic three-dimensional stimuli by 3-month-old infants. Infancy, 16, 435-445.

Needham, A., \& Libertus, K. (2011). Embodiment in early development. Wiley Interdisciplinary Reviews: Cognitive Science, 2, 117-123.

Newcombe, N. S., \& Frick, A. (2010). Early education for spatial intelligence: Why, what, and how. Mind, Brain, and Education, 4, 102-111.

Piaget, J. (1952). The origins of intelligence in children (M. Cook, Trans.). New York: International Universities Press. (Original work published 1936).

Piaget, J., \& Inhelder, B. (1956). The child's conception of space (F. J. Langdon \& J. L. Lunzer, Trans.). London: Routledge and Kegan Paul. (Original work published 1948).

Piaget, J., \& Inhelder, B. (1971). Mental imagery in the child; a study of the development of imaginal representation (P. A. Chilton, Trans.). New York: Basic. (Original work published 1966). 
Quinn, P. C., \& Liben, L. S. (2008). A sex difference in mental rotation in young infants. Psychological Science, 19, 1067-1070.

Rochat, P., \& Hespos, S. J. (1996). Tracking and anticipation of invisible spatial transformation by 4- to 8-month-old infants. Cognitive Development, 11, 3-17.

Schwarzer, G., Freitag, C., Buckel, R., \& Lofruthe, A. (2012). Crawling is associated with mental rotation ability by 9 -month-old infants. Infancy. DOI: 10.1111/j.15327078.2012.00132.x

Shepard, R. N., \& Metzler, J. (1971). Mental rotation of three-dimensional objects. Science, $171,701-703$.

Wilson, M. (2002). Six views of embodied cognition. Psychonomic Bulletin and Review, 9, 625-636.

Zwaan, R. A. (1999). Embodied cognition, perceptual symbols, and situation models. Discourse Processes, 28, 81-88. 


\section{Appendix: Parent questionnaire assessing infants' motor development.}

For the following motor abilities, parents were asked (in German):

a) whether the child showed this behavior and

b) since when (at which age) the child showed the behavior.

My child...

... tries to reach for objects.

... tries to grasp objects.

... clasps objects with his/her hand.

... lifts an object unrequestedly.

... turns an object .

... reaches for small objects, using the so-called precision grip

(questionnaire included a picture showing a precision grip).

... hits two objects together during playing.

... inserts one object into another one.

... handles more than one object.

$\ldots$ is able to drink out of a glass on its own.

$\ldots$ holds the glass and tilts it.

... turns on its belly and back.

... rises into a half-seated position.

... sits with assistance.

... sits on its own without assistance.

... sits and plays.

... crawls with its belly touching the floor.

... crawls on hands and knees (without its belly touching the floor).

... moves forward using another possibility - which one?

... stands up with assistance.

... stands up on its own.

... stands with assistance.

... stands freely.

... walks a few steps with assistance.

... walks on its own. 
Table 1

Motor abilities showing significant correlations (Spearman's Rho; $p<.05$ ) with performance in the mental rotation task (difference score), and the same correlations after controlling for age.

\begin{tabular}{|c|c|c|c|c|}
\hline & $\begin{array}{c}\text { Spearman's } \\
\text { Rho }\end{array}$ & $p$ & $\begin{array}{c}\text { Rho Controlled } \\
\text { for Age }\end{array}$ & $p$ \\
\hline Reaching for objects & 0.371 & 0.020 & 0.174 & 0.295 \\
\hline Grasping objects & 0.414 & 0.009 & 0.248 & 0.134 \\
\hline Clasping objects & 0.370 & 0.020 & 0.214 & 0.197 \\
\hline Lifting objects & 0.401 & 0.011 & 0.251 & 0.128 \\
\hline Turning objects & 0.349 & 0.029 & 0.181 & 0.277 \\
\hline Tilting glass & 0.364 & 0.021 & 0.397 & $0.012 *$ \\
\hline Rising to half-seated position & 0.348 & 0.028 & 0.217 & 0.185 \\
\hline Crawling on hands \& knees & 0.400 & 0.011 & 0.253 & 0.121 \\
\hline Standing up with assistance & 0.445 & 0.004 & 0.356 & $0.026 *$ \\
\hline Standing freely & 0.329 & 0.038 & 0.190 & 0.247 \\
\hline Standing with assistance & 0.462 & 0.003 & 0.384 & $0.016 *$ \\
\hline Walking with assistance & 0.490 & 0.001 & 0.399 & $0.012 *$ \\
\hline
\end{tabular}




\section{Figure Captions}

Figure 1. Front (top row) and back sides (bottom row) of the symmetrical object ("T") used in the familiarization events, and the asymmetrical objects ("p" and "q") used in the test events of both experiments.

Figure 2. Sequence of a test event (from top to bottom) with examples of a possible (left) and an impossible (right) outcome. Dashed lines indicate the trajectory of the stimulus object.

Figure 3. Mean looking times at possible and impossible test events in 8- and 10-month-old infants. Error bars represent standard errors.

Figure 4. Mean looking times at possible and impossible test events by outcome orientation, for (a) 8-month-olds and (b) 10-month-olds.

Figure 5. Mean looking times at possible and impossible test events by locomotor status. 
Figure 1
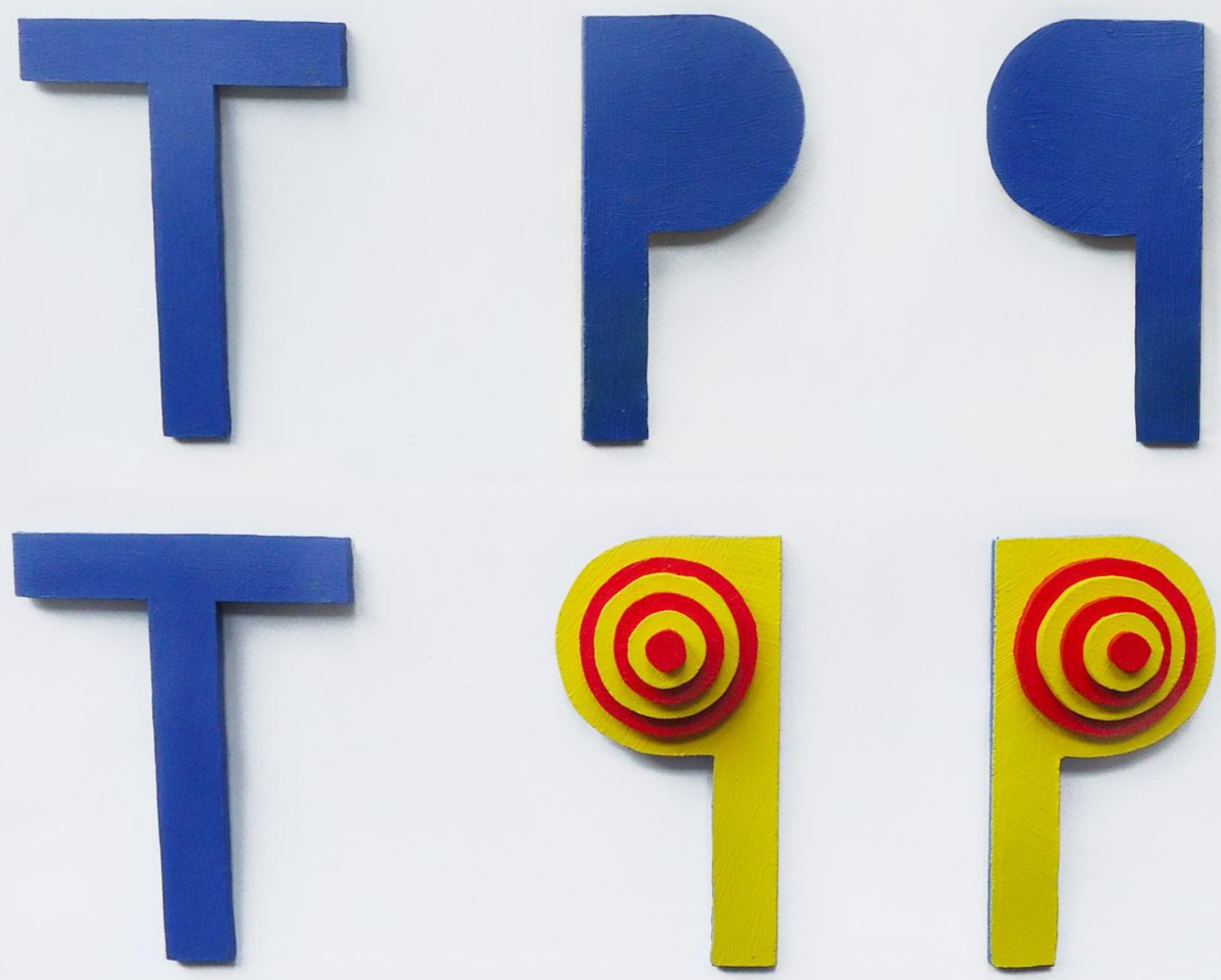
Figure 2

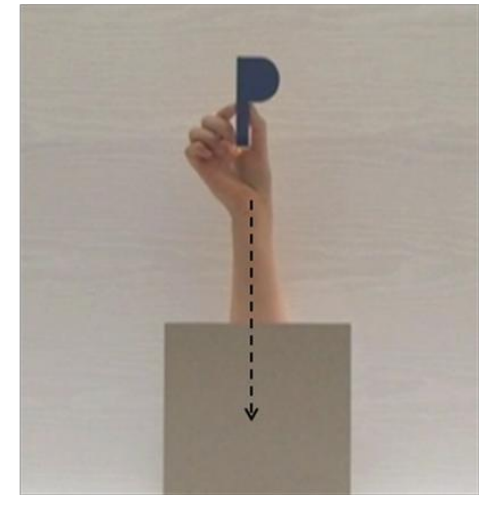

Possible Test Event (e.g., $180^{\circ}$ )

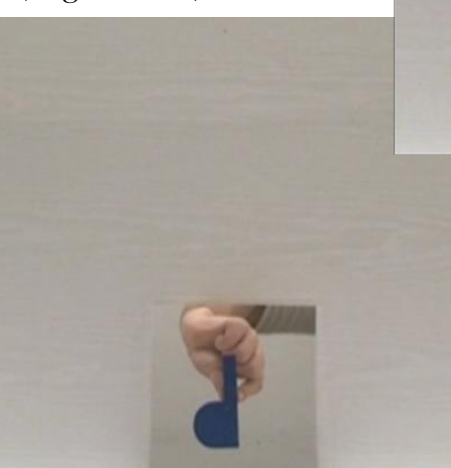

Impossible Test Event (e.g., $45^{\circ}$ ) 
Figure 3

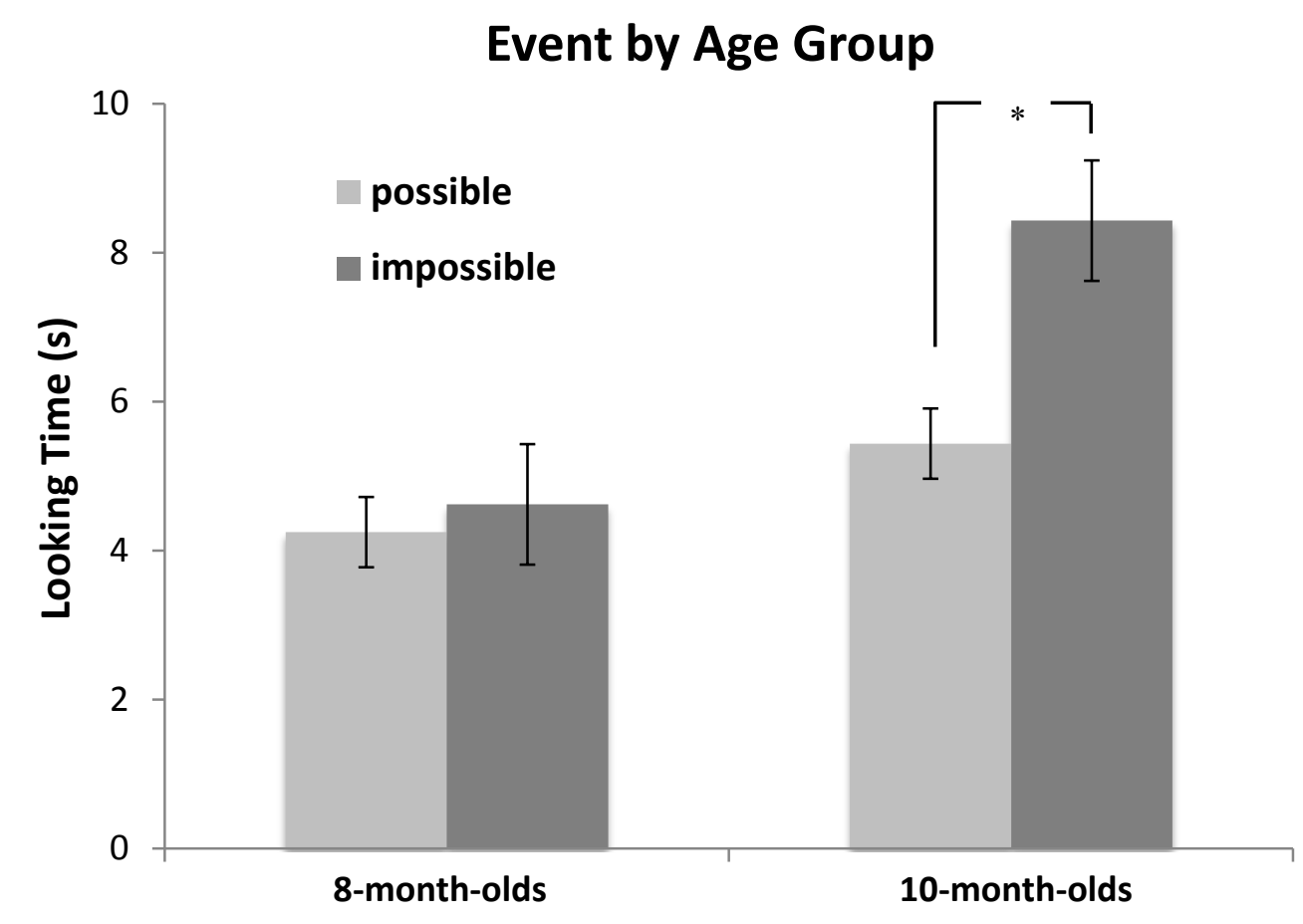


Figure 4

a) 8-Month-Old Infants

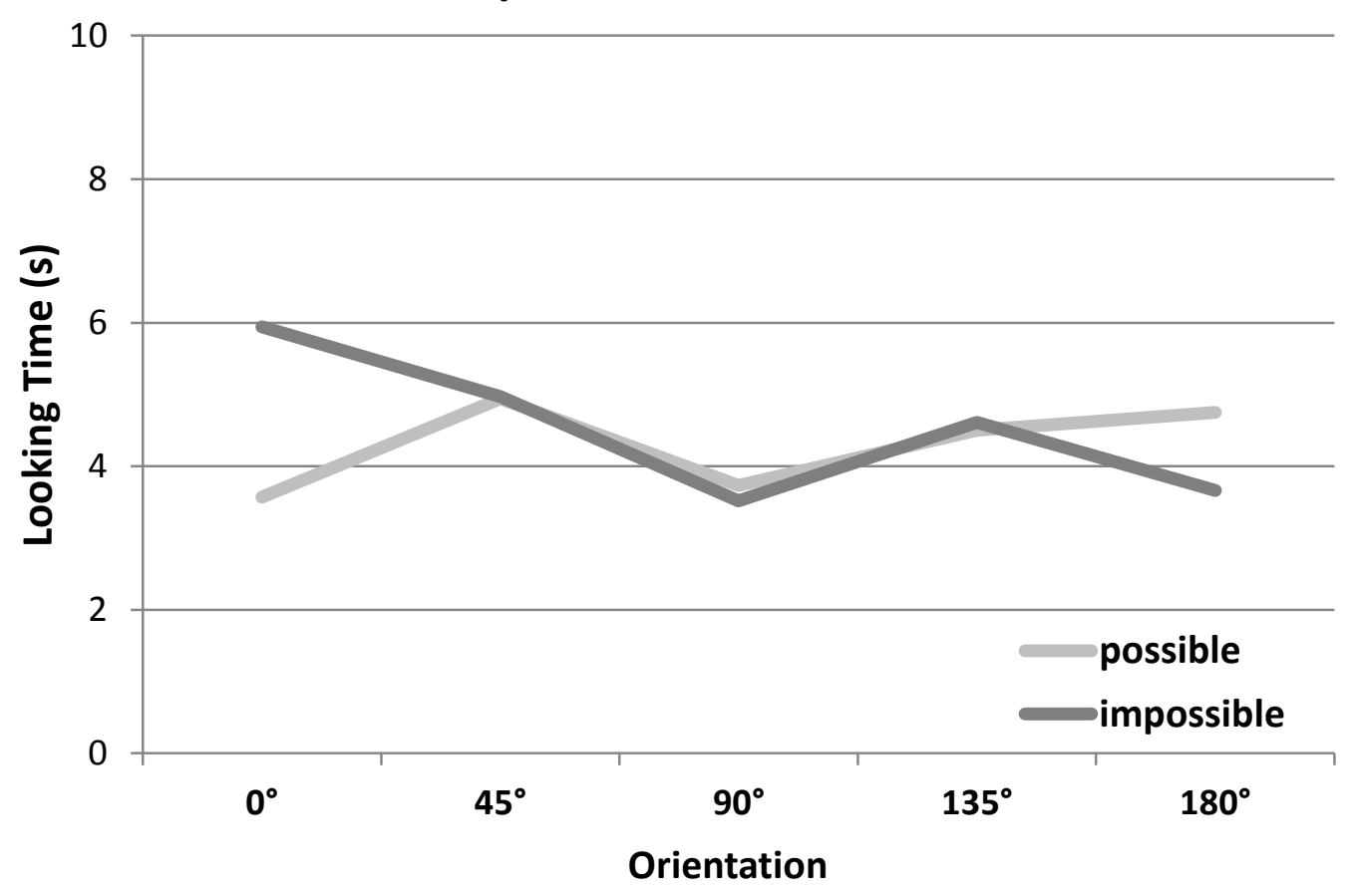

b) 10-Month-Old Infants

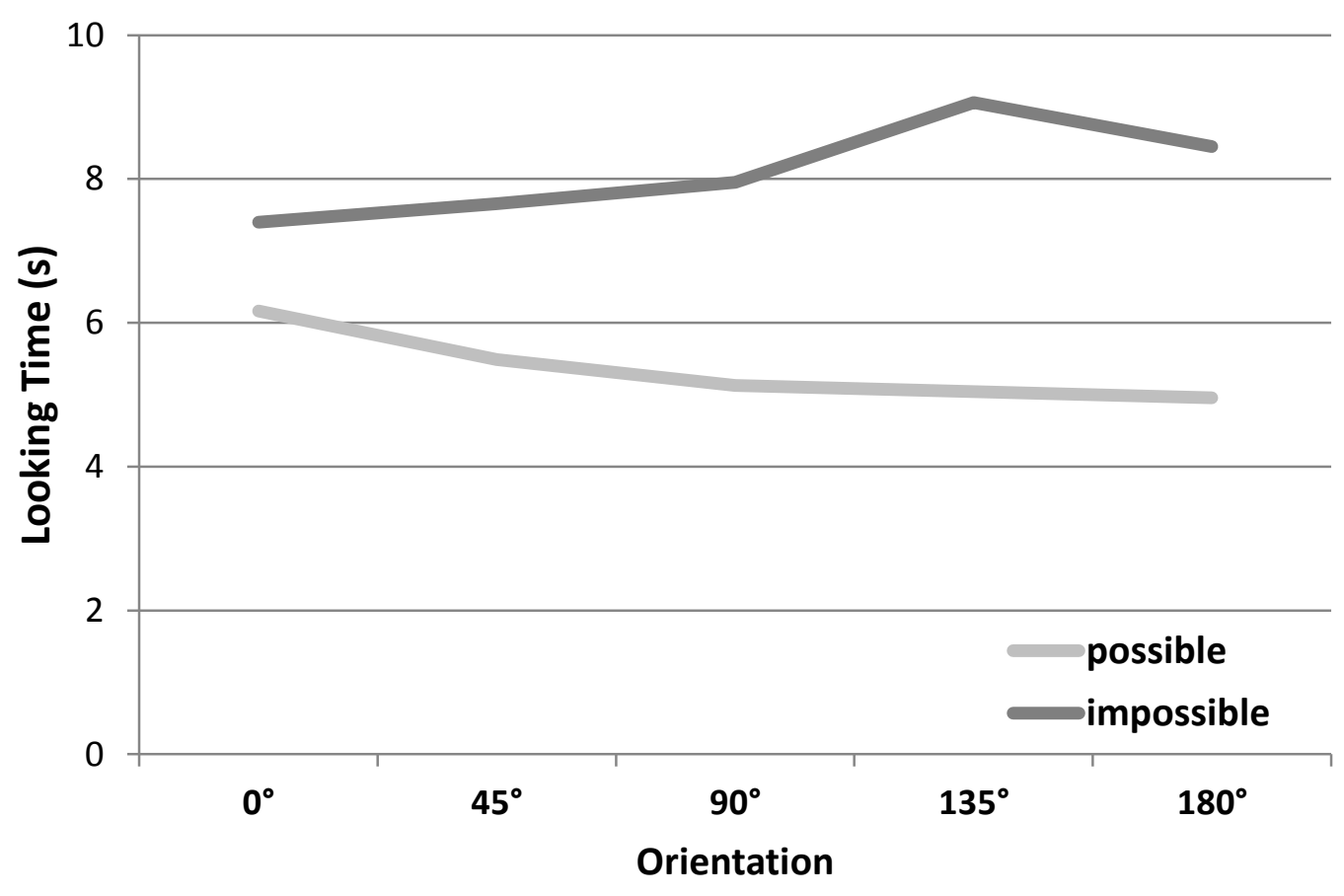


Figure 5

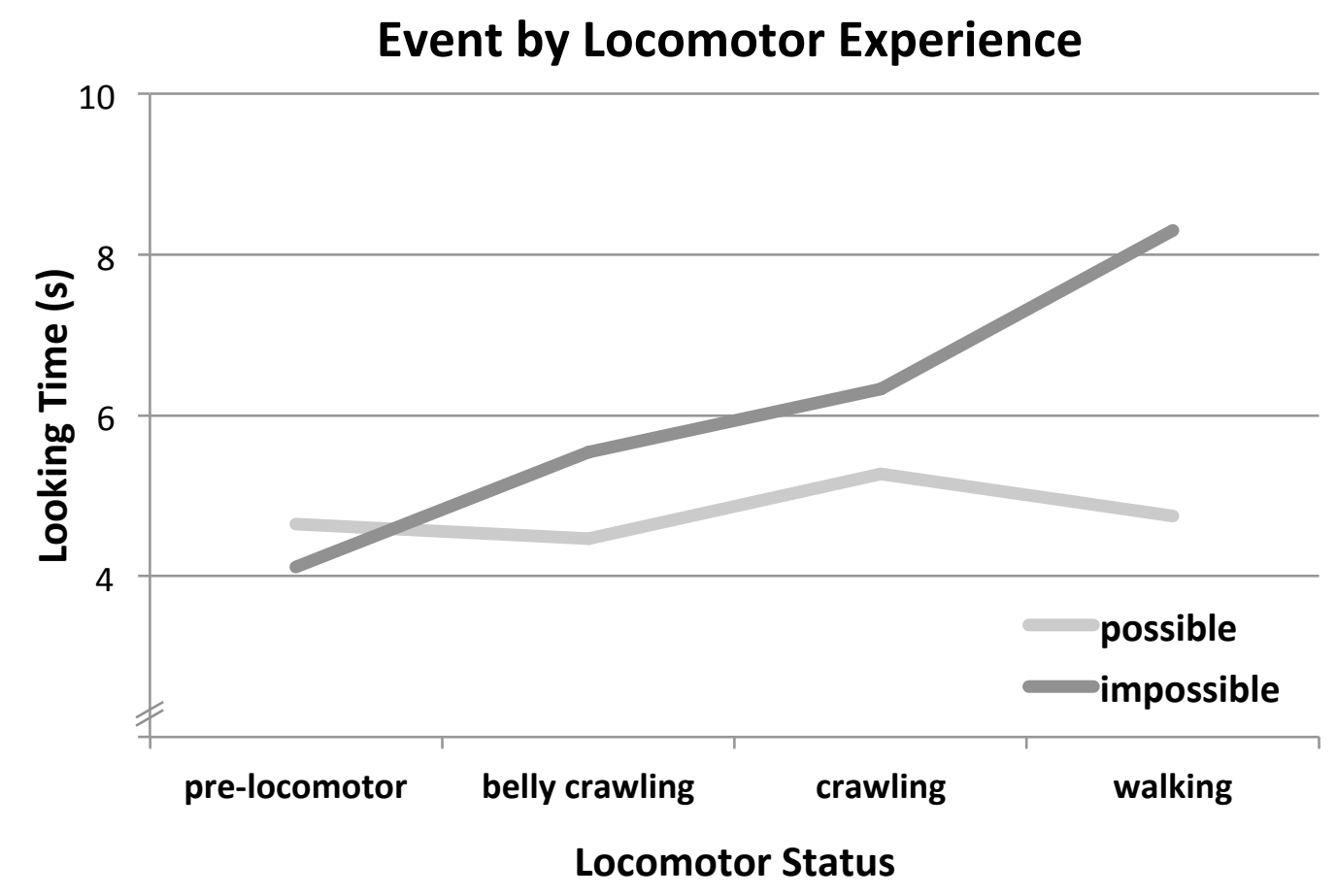

